\title{
INFLUENCIA DE LA VARIABILIDAD EDAFICA EN LA PRODUCCION DE BIOMASA DEL CULTIVO DE LA UÑA DE GATO Uncaria Tomentosa (Willd) D.C. EN LA CUENCA DEL RIO AGUAYTIA, REGION UCAYALI -PERU
}

\section{EDAPHIC VARIABILITY INFLUENCE ON THE BIOMASS PRODUCCTION OF CAT'CLAW Uncaria tomentosa (Willd) D.C. IN THE AGUAYTIA WATERSHED, UCAYALI, PERU}

\author{
Teresa E. Mechán ${ }^{1}$, Julio Alegre ${ }^{2}$ y Gilberto Domínguez ${ }^{3}$
}

\begin{abstract}
Resumen
En Mayo 2003 se instalaron cuatro plantaciones de uña de gato Uncaria tomentosa (Willd) D.C a lo largo de la Cuenca del Río Aguaytia en Ucayali Perú. Las plantas correspondieron al clon 11, propagadas in vitro, y procedentes del Instituto de Biotecnología (IBT) de la Universidad Nacional Agraria La Molina (UNALM). El estudio se basó en la determinación de la influencia edáfica en la producción de biomasa de esta especie como cultivo. Las áreas experimentales se encuentran a diferentes niveles altitudinales desde los 136 hasta los $884 \mathrm{msnm}$.

En Junio 2005 se realizó la primera poda y el muestreo de suelos a dos profundidades, de 0 a 15 y de 15 a $30 \mathrm{~cm}$ del suelo.La textura arcillosa en los suelos fueron las que correlacionaron mejor con la producción de la biomasa del clon; siendo las ecuaciones más representativas: a) Biomasa = 27.329(\% Arena) - 51.875 y b) Biomasa = -32.247(arcilla) - 2169.8. Los indicadores mas significativos de la fertilidad del suelo en el proceso de adaptación de esta especie como cultivo fueron: los contenidos de fósforo (P), materia orgánica (M.O.), nitrógeno $(\mathrm{N})$ total, saturación de aluminio (\% $\mathrm{Al})$, potasio $(\mathrm{K})$, magnesio $(\mathrm{Mg})$ y el $\mathrm{pH}$.
\end{abstract}

Palabras Claves: suelos ácidos, fertilidad, uña de gato, variabilidad del suelo.

\begin{abstract}
Four cat'claw Uncaria tomentosa (Willd) D.C plantations were installed in May 2003 along the Aguaytia river basin in Ucayali, Peru. The plants corresponded to clone 11, in vitro cultured, at the Institute of Biotechnology (IBT) of the Agrarian University (UNALM). The experiment was based on the determination of the edafo-climatic influence on the behavior of this species as a crop. The experimental areas were at different altitude levels that range from 884 to 136 m.a.s.l. First pruning was performed on June 2005 and soil samples were taken from 0 to 15 and from 15 to $30 \mathrm{~cm}$ depths. Soil clay content was correlated significantly with the clone's biomass production and the most representative equations were a) Biomass $=27.329$ ( $\%$ Sand) -51.875 and b) Biomass $=-32.247$ (clay) - 2169.8. Most significant indicators of soil fertility in the process of adaptation of this species as crop were the phosphorus $(\mathrm{P})$, organic matter $(\mathrm{OM})$, total nitrogen $(\mathrm{N})$, aluminum saturation (\%Al), potassium $(\mathrm{K})$, magnesium $(\mathrm{Mg})$ content and $\mathrm{pH}$.
\end{abstract}

Key words: Acid soils, fertility, Uncaria tomentosa (Willd) D.C., soil variability.

\section{Introducción}

Dentro de la diversidad de plantas medicinales que posee la amazonía, la uña de gato Uncaria tomentosa (Willd) DC, especie nativa del Perú, ha sido utilizada desde tiempos inmemoriales por diversos grupos étnicos de la selva en el tratamiento de sus diversas enfermedades, conocimiento y recurso valioso que ha podido ser preservado sólo de generación en generación, llegando en la última década ha trascender en el campo de la medicina científica (Flores, 1995; Domínguez, 1997; Zavala, 1995; Obregón, 1994; Quinteros, 2001). Además, esta especie ha logrado ingresar al mercado nacional e internacional en forma muy acelerada, dado que el $80 \%$ de la población mundial recurre a la medicina tradicional para atender sus necesidades primarias médicas, arriesgando su abastecimiento a futuro (Gómez, 2004). Los suelos de la amazonía peruana son muy frágiles por sus bajos niveles de nutrientes y materia orgánica y alta saturación de aluminio que es tóxica para muchas plantas y el comportamiento de algunas plantas nativas va a depender de la tolerancia a los niveles tóxicos de la saturación de aluminio y a bajos niveles de nutrientes disponibles (Alegre et al., 1988; Ocampo, 1997). La textura y la distribución de partículas de los suelos son las que regulan los procesos de movimiento del agua en el suelo y la capacidad retentiva de agua influenciando en la solubilidad y disponibilidad de los nutrientes para las plantas (Zavaleta, 1992; Alegre et al., 1988) 
Este estudio tuvo por objetivos: a) determinar los efectos de la variabilidad edáfica en la producción de la biomasa en plantaciones de uña de gato ubicadas a lo largo de la cuenca hidrológica del Aguaytia y b) Establecer índices de textura del suelo en relación con los niveles nutricionales para un mejor establecimiento del cultivo de uña de gato Uncaria tomentosa (Willd) D.C.

\section{Materiales y Métodos \\ Descripción del Sitio:}

Las 4 áreas experimentales, fueron establecidas desde mayo de 2003, ubicados dentro de un rango de $8^{\circ} 23^{\prime}-9^{\circ} 8^{\prime}$ de Latitud Sur, y $74^{\circ} 38^{\prime}-75^{\circ} 46^{\prime}$ de Longitud Oeste, y a diferentes niveles altitudinales que fluctuaron desde los 136 hasta los $884 \mathrm{msnm}$. Las parcelas A, B,C y D, de $15 \mathrm{~m}^{2}$ cada una, se ubicaron a lo largo de la carretera Federico Basadre en el 12.4 km, 98 km, 152 km y 203 km respectivamente. El clima es húmedo tropical, con temperatura promedio de $26{ }^{\circ} \mathrm{C}$. La precipitación muestra cierta variación de estacionalidad y de intensidad dentro de la cuenca del río Aguaytia señalándose una precipitación promedio anual de $2200 \mathrm{~mm}$.

\section{Evaluaciones:}

En Junio 2005 se inició la realización de la primera poda de las 4 plantaciones del clon 11 de la especie Uncaria tomentosa, seguidamente a la poda, se efectuó la separación manual de las hojas y ramas a fin de facilitar su manipulación para el secado y evitar la alteración de sus propiedades bioquímicas y principios activos medicinales. La biomasa seca se determinó después de secar las plantas en estufas de circulación de aire a temperaturas no mayores de 75 ${ }^{\circ} \mathrm{C}$. Asimismo se realizó el muestreo de suelos, tomándose para ello 10 puntos referenciales en cada parcela y a dos profundidades, de 0 a 15 y de 15 a 30 $\mathrm{cm}$.

Finalizada la etapa de campo, tanto las muestras de suelo como las de biomasa fueron trasladadas al Laboratorio de Análisis de suelos y de aguas de la Universidad Nacional Agraria la Molina (UNALM) para la realización del análisis de caracterización de los suelos como textura, $\mathrm{pH}$, materia orgánica (MO), fósforo $(\mathrm{P})$, potasio $(\mathrm{K})$, calcio $(\mathrm{Ca})$, magnesio $(\mathrm{Mg}) \mathrm{y}$ saturación de $\mathrm{Al}$ (\%Al), de acuerdo a las metodologías descritas por Bazán (1996).

Se elaboraron correlaciones simples de cada una de las partículas de arena, limo y arcilla del suelo así como los nutrientes que corresponden a sus diferentes clases texturales con la respectiva biomasa producida en cada parcela.

\section{Resultados y Discusión \\ Análisis de producción}

El análisis estadístico se realizó eliminando 3 plantas de las 10 tomadas al inicio por cada parcela debido al excesivo daño que se observó por las hormigas cortadoras y a cierta variabilidad entre plantas los cuales mostraron coeficientes de variación mayores al $100 \%$, pero mediante una transformación logarítmica de los promedios se ajustó a una curva normal de distribución bajándose el coeficiente de variación al 12\%.

Tabla 1. Producción promedio de la biomasa de uña de gato en cada parcela con su respectiva altitud, $\mathrm{pH}$, clase textural y rango de partículas.

\begin{tabular}{lllllc} 
Parcela & Altitud & $\begin{array}{c}\text { Clase } \\
\text { textural }\end{array}$ & $\begin{array}{c}\text { Partículas } \\
(\%)\end{array}$ & pH & $\begin{array}{c}\text { Peso de } \\
\text { biomasa (gr) }\end{array}$ \\
\hline A & \multirow{2}{*}{136} & Franco & $\begin{array}{l}46-52 / 30-44 \\
/ 8-18\end{array}$ & 3.8 & 47.98 \\
B & 236 & Arcilloso & $\begin{array}{l}12-26 / 22-34 \\
/ 44-62\end{array}$ & 6.1 & $541.76^{*}$ \\
C & \multirow{2}{*}{305} & $\begin{array}{l}\text { Franco } \\
\text { arcilloso }\end{array}$ & $\begin{array}{l}36-72 / 16-34 \\
/ 10-38\end{array}$ & 4.0 & $453.28^{*}$ \\
D & \multirow{2}{*}{885} & $\begin{array}{l}\text { Franco } \\
\text { arcilloso }\end{array}$ & $\begin{array}{l}30-42 / 30-40 \\
/ 28-32\end{array}$ & 5.2 & $985.48^{*}$ \\
\hline
\end{tabular}

Se observa que la mayor producción de biomasa por planta se dio en la parcela D, a los $885 \mathrm{msnm}$, siendo ésta significativamente superior a la producción de la parcela A, del km 12.4, el cual mostró los más bajos rendimientos.

El pH del suelo de la parcela $\mathrm{D}$ con la mejor producción fue de 5.2 mientras que la más baja presentó un pH de 3.8 y, como se observa más adelante, también los niveles de potasio (K) fueron muy bajos en esta zona.

La textura también fue diferente, presentó una textura ideal franco arcillosa el cual permite una mayor disponibilidad de agua y una mejor aireación facilitando la absorción de los nutrientes.

\section{Coeficientes de Correlación Simple}

Se realizó una primera correlación entre la biomasa de las plantas evaluadas con las diferentes partículas del suelo en forma conjunta de las 4 zonas, sin embargo el análisis mostró muy bajo nivel de significación. Por lo que al realizar una segunda correlación, más detallada, por zona y a cada profundidad si se obtuvo diferencias altamente significativas. Esto mismo se obtuvo en un trabajo realizado en la zona de Ucayali para la especie de Bractris gasipaes (Mendoza, 2005)

En la Tabla 2 se puede observar que en la capa superficial tanto las partículas de arena y arcilla muestran ambas una asociación del 13\% con respecto a la biomasa. 
Tabla 2. Coeficientes de correlación simple entre las partículas del suelo y la producción de biomasa de uña de gato en cada parcela y a dos profundidades del suelo (0-15 y 15-30 cm).

\begin{tabular}{lcccccc} 
& \multicolumn{6}{c}{ Partículas del Suelo } \\
\cline { 2 - 7 } & \multicolumn{2}{c}{ Arena } & \multicolumn{2}{c}{ Limo } & Arcilla \\
\cline { 2 - 7 } & $0-15$ & $15-30$ & $0-15$ & $15-30$ & $0-15$ & $15-30$ \\
\hline Parcela & $(\mathrm{cm})$ & $(\mathrm{cm})$ & $(\mathrm{cm})$ & $(\mathrm{cm})$ & $(\mathrm{cm})$ & $(\mathrm{cm})$ \\
\hline $\begin{array}{l}\text { A } \\
\mathrm{km} 12.4\end{array}$ & $0.64^{* *}$ & $0.56 \mathrm{~ns}$ & $0.23 \mathrm{~ns}$ & $0.53 \mathrm{~ns}$ & $0.40 \mathrm{~ns}$ & $0.33 \mathrm{~ns}$ \\
\hline $\begin{array}{l}\text { B } \\
\mathrm{km} \mathrm{98}\end{array}$ & $0.95^{*}$ & $0.61 \mathrm{~ns}$ & $-0.87^{*}$ & $0.81^{*}$ & $-0.93^{*}$ & $0.05 \mathrm{~ns}$ \\
\hline $\begin{array}{l}\mathrm{C} \\
\mathrm{km} \mathrm{152}\end{array}$ & $0.22 \mathrm{~ns}$ & $0.62 \mathrm{~ns}$ & $0.40 \mathrm{~ns}$ & $0.70^{*}$ & $0.07 \mathrm{~ns}$ & $0.38 \mathrm{~ns}$ \\
\hline $\begin{array}{l}\mathrm{D} \\
\mathrm{km} \mathrm{203}\end{array}$ & $0.37 \mathrm{~ns}$ & $0.03 \mathrm{~ns}$ & $0.14 \mathrm{~ns}$ & $0.21 \mathrm{~ns}$ & $0.57^{* *}$ & $0.16 \mathrm{~ns}$ \\
\hline
\end{tabular}

$(*)$ : Significativos $(\mathrm{P}<=0.01)$; (ns) no significativo.

El análisis de correlación para las diferentes partículas del suelo en forma individual determinó que los mayores coeficientes de correlación, altamente significativos, fueron en la parcela B (km 98) a la profundidad de 0-15 cm para arena, limo y arcilla, y para la profundidad 15-30 cm solo para limo (Tabla 2).

En la Parcela A (km 12.4) de clase textural Franco, sólo se observó valores altos de correlación en la partícula de arena superficial y sub-superficial. Por tanto; en los primeros $15 \mathrm{~cm}$ de profundidad del suelo la biomasa se asocia en un $64.0 \%$ con la fracción de arena y las otras partículas se asocian en muy bajas cantidades.

En la Parcela B, la clase textural es arcillosa, y en los primeros $15 \mathrm{~cm}$. del suelo, la biomasa se asocia en un 95\%, 87 \% y $93 \%$ con las partículas arena, limo y arcilla respectivamente (Figura 2). En el rango de 15 a $30 \mathrm{~cm}$ de profundidad, sólo la partícula limo presentó la más alta asociación (81\%).

Para la parcela C (km 152), en los primeros $15 \mathrm{~cm}$ del suelo, solo la partícula de limo obtuvo un $40 \%$ de asociación, y un 70\% en el rango de 15-30 cm del suelo.

En la parcela D, la clase textural es franco arcillosa, y en los primeros $15 \mathrm{~cm}$ de suelo la biomasa se asoció sólo en $37 \%, 14 \%$ y $57 \%$ con las partículas arena, limo y arcilla respectivamente. (Figura 3).

El coeficiente de determinación más alto se obtuvo para la parcela A del km 12.4, como se observa en la Figura 2. En la siguiente ecuación:

Arena; $\left.\mathrm{y}=3.7857 \mathrm{x}-144.54 \mathrm{R}^{2}=0.4141\right)$

El coeficiente de determinación $\left(\mathrm{R}^{2}=0.4141\right)$ nos muestra que el $41.41 \%$ de la variación de la biomasa está en función de la arena en los primeros $15 \mathrm{~cm}$ del suelo.
Para la parcela B del km 98, como se observa en la Figura 6. En la siguiente ecuación:

Arena: $\mathrm{y}=27.329 \mathrm{x}-51.875 \quad \mathrm{R}^{2}=0.8544$

El coeficiente de determinación $\left(\mathrm{R}^{2}=0.854\right)$ nos muestra que el $85.44 \%$ de la variación de la biomasa está en función de la partícula de arena en los primeros $15 \mathrm{~cm}$ del suelo.

Para la parcela D del km 203 la ecuación de la partícula Arcilla fue:

$$
\mathrm{y}=192.04 \mathrm{x}-4172.2 \text { con una } \mathrm{R}^{2}=0.327
$$

El coeficiente de determinación $\left(\mathrm{R}^{2}=0.327\right)$ nos muestra que el $32.7 \%$ de la variación de la biomasa está en función de la arcilla en los primeros $15 \mathrm{~cm}$ del suelo. Cabe señalar que la clase textural en la parcela B (km 98), es arcillosa, y a pesar de tener una mayor proporción de arcilla en el suelo, su influencia fue relativamente menor $(81.2 \%)$ en relación a la partícula arena, y superior en comparación con la partícula limo, cuya proporción en el suelo es menor que la arcilla, y con una influencia del $75 \%$ en la variación de la biomasa de la uña de gato producida.

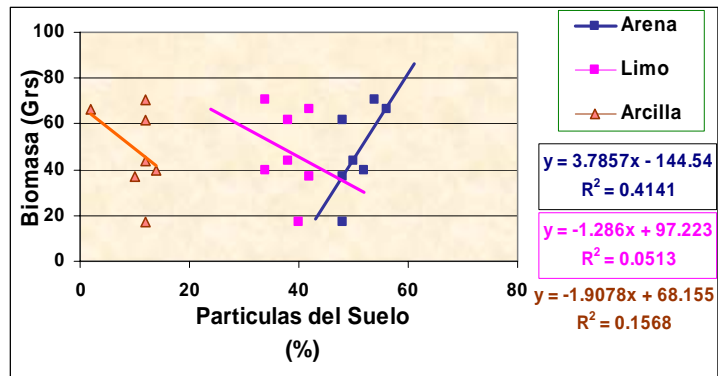

Figura 1. Relación entre las partículas del suelo versus la producción de la biomasa en la parcela A del km 12.4 .

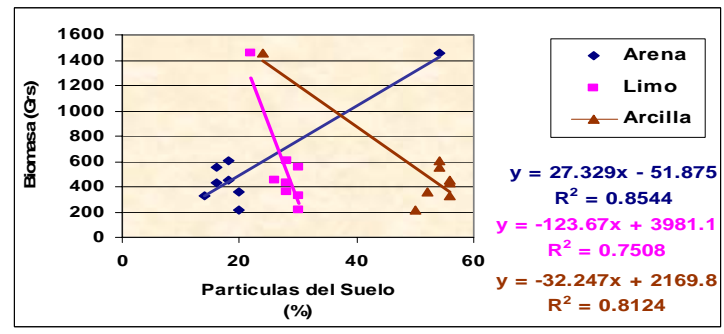

Figura 2. Relación entre las partículas del suelo versus la producción de la biomasa en la parcela B del km 98.

\section{Producción de biomasa versus nutrientes del suelo}

En la Tabla 4 son mostrados los análisis de fertilidad y se puede observar la variabilidad del aporte nutricional del suelo entre los tratamientos y está en función de la distribución de las partículas. Esto determina la mayor o menor capacidad para retener nutrientes manifestándose en el diferente comportamiento de las plantas de uña de gato de acuerdo a la toposecuencia de ubicación de los suelos 
de buen y mal drenaje los cuales están bastantes diferenciados en la carretera Federico Basadre desde su inicio en Pucallpa hasta el km 203.

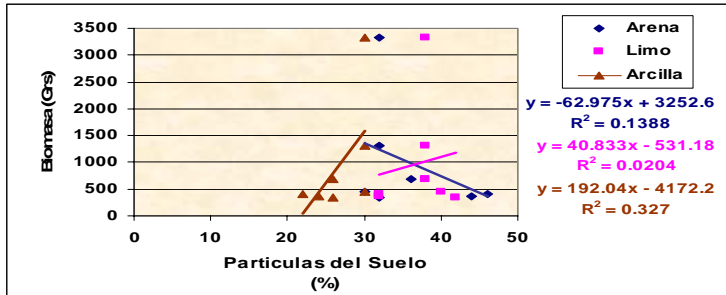

Figura 3. Relación entre las partículas del suelo versus la producción de la biomasa en la parcela D del km 203.

Tabla 4. Producción promedio de la biomasa para los diferentes sitios de plantación con uña de gato en relación con el contenido promedio de nutrientes del suelo para cada profundidad $(0-15$ y $15-30 \mathrm{~cm})$

\begin{tabular}{lllllllll}
\hline Parcela & $\begin{array}{l}\mathrm{M} . \mathrm{O} \\
(\%)\end{array}$ & $\mathrm{pH}$ & $\begin{array}{l}\mathrm{N} \\
\text { total } \\
(\%)\end{array}$ & $\begin{array}{l}\mathrm{P} \\
(\mathrm{ppm})\end{array}$ & $\begin{array}{l}\mathrm{K} \\
(\mathrm{ppm})\end{array}$ & $\begin{array}{l}\mathrm{Ca} \\
(\mathrm{meq} / 100 \mathrm{gr})\end{array}$ & $\begin{array}{l}\mathrm{Mg} \\
\text { (meq/100gr) }\end{array}$ & $\begin{array}{l}\text { Biomasa } \\
\text { /planta } \\
\text { (gr.) }\end{array}$ \\
\hline $\mathrm{A}$ & & & & & & & & \\
$0-15$ & 2.06 & 3.8 & 0.10 & 6.9 & 22.7 & 1.47 & 0.33 & 47.98 \\
$15-30$ & 1.15 & 3.7 & 0.06 & 3.6 & 11.4 & 0.93 & 0.15 & \\
B & & & & & & & & \\
$0-15$ & 4.6 & 6.1 & 0.36 & 6.8 & 287.6 & 40.39 & 4.59 & 541.76 \\
$15-30$ & 2.0 & 6.1 & 0.16 & 3.4 & 159.6 & 39.4 & 3.79 & \\
C & & & & & & & & \\
$0-15$ & 3.2 & 4.1 & 0.20 & 5.9 & 238.5 & 2.5 & 1.22 & 453.28 \\
$15-30$ & 2.0 & 3.9 & 0.15 & 3.8 & 178.8 & 1.27 & 0.70 & \\
D & & & & & & & & \\
$0-15$ & 3.7 & 5.3 & 0.19 & 8.1 & 112.0 & 13.73 & 1.39 & 985.48 \\
$15-30$ & 1.9 & 5.2 & 0.11 & 4.7 & 69.8 & 10.8 & 1.14 & \\
\hline
\end{tabular}

Tabla 5. Correlaciones obtenidas en relación a la producción de biomasa versus el contenido de nutrientes en el suelo de cada sitio evaluado.

\begin{tabular}{|c|c|c|c|c|c|c|c|c|}
\hline \multirow{3}{*}{ Variable } & \multicolumn{2}{|c|}{ Parcela A } & \multicolumn{2}{|c|}{ Parcela B } & \multicolumn{2}{|c|}{ Parcela C } & \multicolumn{2}{|c|}{ Parcela D } \\
\hline & \multicolumn{2}{|c|}{$\begin{array}{l}\text { Profundidad } \\
\qquad \mathrm{cm}\end{array}$} & \multicolumn{2}{|c|}{$\begin{array}{l}\text { Profundidad } \\
\text { cm }\end{array}$} & \multicolumn{2}{|c|}{$\begin{array}{c}\text { Profundidad } \\
\text { cm }\end{array}$} & \multicolumn{2}{|c|}{$\begin{array}{l}\text { Profundidad } \\
\text { Cm }\end{array}$} \\
\hline & $0-15$ & $15-30$ & $0-15$ & $15-30$ & $0-15$ & $15-30$ & $0-15$ & $15-30$ \\
\hline $\mathrm{pH}$ & $0.59 * *$ & 0.14 & $0.49 * *$ & $0.77 *$ & 0.15 & 0.23 & $0.48 * *$ & 0.06 \\
\hline M.O & 0.40 & 0.32 & $0.87 *$ & $0.73 *$ & 0.21 & $0.65^{* *}$ & $0.70 * *$ & -0.35 \\
\hline $\mathrm{N}$ & $0.83 *$ & 0.15 & $0.81 *$ & 0.55 & 0.21 & 0.56 & 0.34 & -0.26 \\
\hline $\mathrm{P}$ & 0.40 & $0.46^{* *}$ & $0.95 *$ & 0.38 & 0.50 & $0.53^{* *}$ & $0.92 *$ & -0.57 \\
\hline K & 0.37 & 0.10 & -0.47 & $0.68 * *$ & -0.16 & 0.30 & 0.20 & $0.72 *$ \\
\hline $\mathrm{Ca}$ & 0.51 & 0.37 & -0.11 & $-0.77^{*}$ & 0.51 & $0.69 * *$ & 0.03 & 0.55 \\
\hline $\mathrm{Mg}$ & $0.67 * *$ & $0.57 * *$ & $0.63^{* *}$ & $0.52 * *$ & $0.70 * *$ & $0.90 *$ & 0.42 & $0.58 * *$ \\
\hline Sat. Al & $-0.75^{*}$ & -0.37 & $0.64 * *$ & $0.57 * *$ & -0.44 & $0.57 * *$ & -0.18 & -0.39 \\
\hline $\begin{array}{l}\text { Sat. } \\
\text { Bases }\end{array}$ & 0.32 & 0.10 & $0.60 * *$ & $0.78 *$ & $0.62 * *$ & 0.47 & 0.21 & -0.16 \\
\hline
\end{tabular}

$(*)$ Altamente significativos $(\mathrm{P}<=0.01)$.

(**) Medianamente significativos.
La producción promedio obtenido de la biomasa de la uña de gato se obtuvo en el siguiente orden de mayor a menor; 985.48 grs, 541.76 grs, 453.28 grs, y 47.98 grs en los tratamientos D, B, C y A respectivamente. La mayor producción de biomasa se dio en la parcela $\mathrm{D}$, esto debido por los mayores contenidos de $\mathrm{P}$ disponible en el suelo y a un $\mathrm{pH}$ de 5.2, ligeramente ácido. Entonces el clon evaluado al parecer se adapta mejor a un $\mathrm{pH}$ mayor a 4 .

La segunda mayor producción ocurre en la parcela B del km 98 que presentó el mayor porcentaje de materia orgánica, $4.6 \%$ en relación a las demás parcelas. Asimismo se observa que posee el mayor contenido de $\mathrm{N}$ total, $\mathrm{K}$, Ca y $\mathrm{Mg}$ en el suelo y su pH cercano a neutro, pero sus niveles de $\mathrm{P}$ son menores que la parcela $\mathrm{D}$.

La parcela C del km 152 no presentó diferencias significativas en producción de biomasa con la parcela B del km 98 y además sus niveles de nutrientes están bajos en $\mathrm{P}$ y en niveles adecuados para el Ca y Mg.

La parcela A del km 12.4 es la que obtuvo los niveles mas bajos de $\mathrm{K}$, Ca y $\mathrm{Mg}$ y con un $\mathrm{pH}$ 3.8, fuertemente ácido obteniéndose la producción de biomasa mas baja

Las mayores correlaciones obtenidas en relación a la producción de biomasa versus el contenido de nutrientes en el suelo de cada sitio evaluado para las profundidad de $0-15 \mathrm{~cm}$ y de $15-30 \mathrm{~cm}$, fueron para el P, MO, $\mathrm{N}$ total y, saturación de Al (Tabla 5).

La saturación de Aluminio fue un buen indicador que correlacionó con la producción de biomasa para los diferentes sitios en las profundidades superficiales y sub-superficiales y este índice de acidez toma en cuenta para su cálculo los niveles de $\mathrm{Ca}, \mathrm{Mg}, \mathrm{K}, \mathrm{y} \mathrm{Al}$ en relación con el Aluminio del complejo de cambio.

El P y K son macronutrientes muy limitantes en estos suelos y así fue documentado por Alegre et al. (1994) , en el cual manifiestan que los niveles debajo de $10 \mathrm{ppm}$ ya se consideran muy críticos para especies nativas como la uña de gato, sin embargo para cultivos anuales estos límites se encuentran entre 12-15 ppm.

\section{Conclusiones}

1. La variabilidad del suelo influenció la producción de biomasa de la uña de gato.

2. La textura y el contenido de nutrientes en el suelo a lo largo de la Cuenca Hidrológica del Aguaytia, en base a la distribución y nivel de precipitación, influyó en la producción de la biomasa del cultivo de la uña de gato.

3. La textura arcillosa fue la que correlacionó mejor con la producción de biomasa de la uña de gato siendo 
las ecuaciones indicadoras representativas las siguientes:

a) Biomasa $=27.329$ (\% Arena) $-51.875 \mathrm{y}$

b) Biomasa $=-32.247$ (arcilla) -2169.8 .

4. El fósforo, materia orgánica, nitrógeno total, saturación de aluminio, potasio, magnesio y el pH del suelo fueron los indicadores de fertilidad más significativos en el desarrollo de uña de gato como cultivo.

\section{Literatura Citada}

Alegre J.C., Cassel D.K. \& Bandy D.E.. 1988. Effect of Land Clearing Method on Chemical Properties of an Ultisol in the Amazon. Soil Sci.Soc.Am.J. Vol 52. Pág.1283

Alegre J.C., Gichuru W. \& Sanchez P. 1994. Fertilización Fosfórica en Sistemas con bajos insumos en los Trópicos Húmedos del Perú. Terra Vol. 12 No 1. 117123

Bazan R. 1996. Manual para el análisis químico de suelos, aguas, plantas. UNALM. Fundación Perú. Lima. Pág. 17.

Domínguez G.1997. Uña de gato y producción sostenible. UNALM. Pág. 15-33.

Flores Y. 1995. Manual para la producción de plantones de uña de gato del Instituto de Investigación Agraria
(INIA). Estación Experimental A. Von Humbolt. Pucallpa, Perú. 35 pp.(www.monografias.com)

Gomez V. 2004. La Uña de gato Uncaria tomentosa (Willd) D.C. Experiencias para un Manejo Sustentable en la Región Ucayali. Trabajo Profesional. UNALM. Pág.7.

Mendoza P. 2005. Influencia de la textura del suelo en el crecimiento del Pijuayo (Bactris gasipaes) sin espinas, en asociación con una leguminosa (Centrosema macrocarpum) en el Trópico Húmedo de Pucallpa. Tesis Ing. Agron. UNALM. Lima - Perú. Pag. 16.

Obregón V. 1994. Uña de gato: Genero Uncaria. Estudios Botánicos, químicos y farmacológicos de Uncaria tomentosa y Uncaria guianensis. Instituto de Fitoterapia Americano. Lima. Perú. Pág. 162.

Ocampo P.M. 1997. Resultados Preliminares de Investigación Forestal en Shampubales en el Alto Mayo Región San Martín. Otorongo No. 05. Pág. 03-17.

Quinteros B. 2001. Primera Reunión Internacional del Genero Uncaria (Uña de Gato) Iquitos- Perú. pp. 101102.

Zavala C. 1995. Taxonomía, distribución y situación Poblacional del Género Uncaria Scherb en el Perú. Tesis de Ing. Forestal. UNALM. Lima. Perú. Pág.105.

Zavaleta G.A. 1992. Edafología. El Suelo en Relación con la Producción. CONCYTEC. Lima. Perú. Pág. 61-72.

\footnotetext{
${ }^{1}$ Ex-alumna Universidad Nacional Agraria La Molina. Av. La La Molina s/n, la Molina, Lima Perú.

${ }^{2}$ Profesor Principal Facultad de Agronomía Universidad Nacional Agraria La Molina. Av. La La Molina s/n, la Molina, Lima Perú. (jalegre@lamolina.edu.pe)

${ }^{3}$ Profesor Principal Facultad de Ciencias Forestales Universidad Nacional Agraria La Molina. Av. La La Molina s/n, la Molina, Lima Perú.
} 